\title{
Comparative effects of variable ventilation and a stepwise recruitment maneuver on atelectasis and hemodynamics in an experimental model of ARDS
}

\author{
L. Vivona', ${ }^{1,}$ A. Braune ${ }^{2}$, T. Bluth², B. Stender ${ }^{3}$, R. Huhle ${ }^{2}$, T. Koch², \\ M. Gama De Abreu²
}

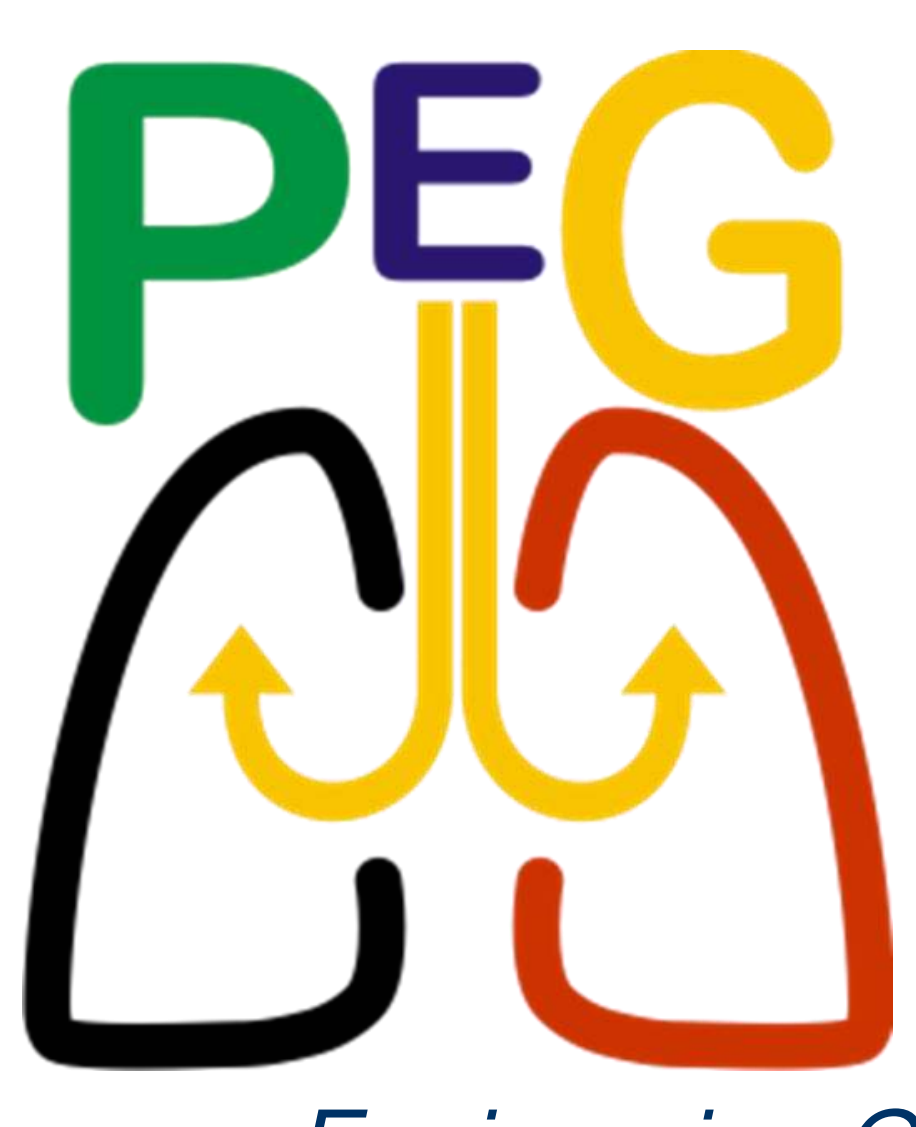

Pulmonary Engineering Group www.peg-dresden.de

Department of Neurosciences, Reproductive and Odonthostomatological Sciences University of Naples "Federico II" - Naples (Italy); 2Department of Anesthesiology and Intensive Care Medicine, Pulmonary Engineering Group, University Hospital Carl Gustav Carus, Technische Universität Dresden - Dresden (Germany);

${ }^{3}$ Drägerwerk AG \& Co KGaA - Lübeck (Germany)

\section{Background:}

Variable volume controlled ventilation (VV) has the potential to reverse atelectasis. We hypothesized that VV is superior to a conventional stepwise recruitment maneuver (RM)

\section{Methods}

*9 pigs (DD24-5131/354/64) were anesthetized and ventilated in airway pressure release ventilation (APRV) mode with positive endexpiratory pressure (PEEP) and driving pressure of $15 \mathrm{cmH} 2 \mathrm{O}$, as well as respiratory rate $(R R)=20 / \mathrm{min}$.

* Four saline lung lavages were performed.

* Lungs recruited in APRV by stepwise increase of PEEP to 45 $\mathrm{cmH} 2 \mathrm{O}(10 \mathrm{cmH} 2 \mathrm{O}$ increments, $\mathrm{RR}=20 / \mathrm{min}$, driving pressure $=15$ $\mathrm{cmH2O}$ ) (stepwise RM). Then, a decremental PEEP trial was conducted in volume-controlled ventilation with $\mathrm{VT}=6 \mathrm{~mL} / \mathrm{kg}$ and $\mathrm{RR}=30 / \mathrm{min}$ to determine the PEEP that resulted in the highest compliance (optimal PEEP).

* Animals were submitted to two recruitment strategies (random sequence, crossover study):

1)stepwise RM followed by 30 min of volume-controlled ventilation with $\mathrm{VT}=6 \mathrm{~mL} / \mathrm{kg}, \mathrm{RR}=30 / \mathrm{min}$ and optimal PEEP;

2) VV, consisting of 30 min volume-controlled ventilation with random variation of $\mathrm{VT}$ (mean $=6 \mathrm{~mL} / \mathrm{kg}$, normal distribution, coefficient of variation of $30 \%), R R=30 / \mathrm{min}$ and optimal PEEP.

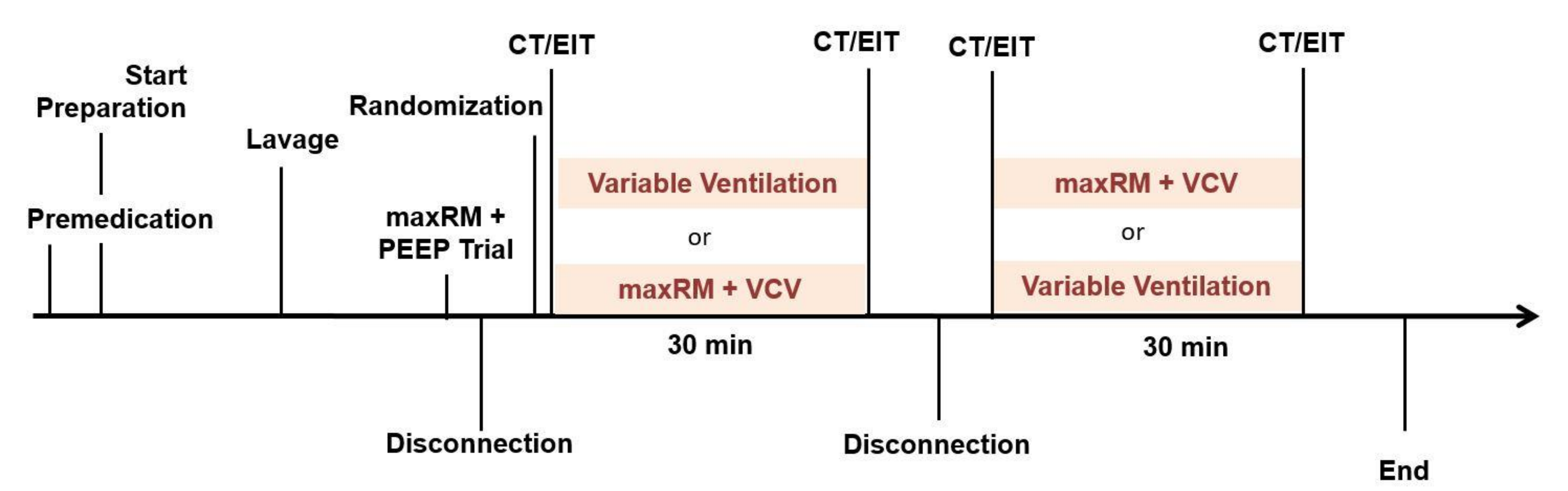

Figure 1 - Time course of interventions and measurements. PEEP, Positive End Expiratory Pressure; RM, Recruitment Maneuver; VCV, Volume Control Ventilation

Before recruitment strategies, animals were disconnected from the ventilator for 30 seconds and volume-controlled mechanical ventilation resumed during 5 minutes with constant $\mathrm{VT}=6 \mathrm{~mL} / \mathrm{kg}$, $\mathrm{RR}=30 / \mathrm{min}$ and optimal PEEP to reset lung history.

I:E ratio and FIO2 were kept constant at 1:1 and 1.0, respectively.

Before and after each recruitment strategy, lung aeration was assessed by computed tomography, and the center of aeration along the ventral-dorsal gradient was determined (Figure2).

\section{Results}

* Both recruitment strategies increased the amount of normally aerated tissue, mainly in dorsal zones (Figure 5), and decreased the amount of poorly and non-aerated lung tissue (Figure 2 and Figure 3)

* More pronounced reduction of non-aerated lung tissue with VV than maxRM (Figure 3)

* The stepwise RM, but not VV, resulted in significant reduction of mean arterial (Figure 4) and increase of heart rate (111 [80...111] to 121 [106 ...131] bpm, $\mathrm{P}=0.004$, median [1st ...3rd quartile]) * Shift of center of aeration in both groups along the ventro-dorsal gradient

* No influence of recruitment strategies on distribution of relative perfusion along the ventro-dorsal gradient (Figure 5)

* Improved $\mathrm{PaO} 2$ and Compliance in both groups (Figure 6)

\section{Conclusion}

In this model of ARDS in pigs, VV more effectively reduced nonaerated lung tissue, with less effects on hemodynamics as compared to a stepwise RM. Head: Prof. Dr. M. Gama de Abreu

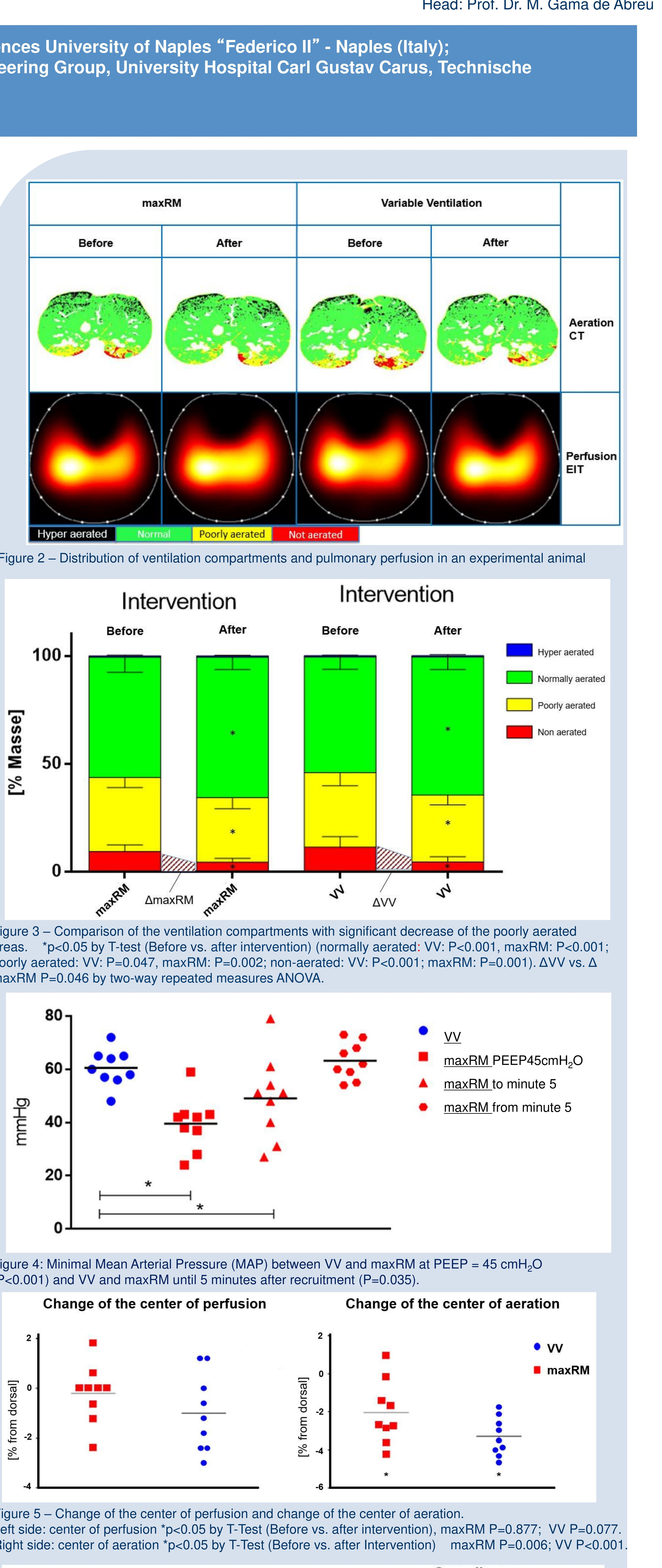

Figure 2 - Distribution of ventilation compartments and pulmonary perfusion in an experimental animal

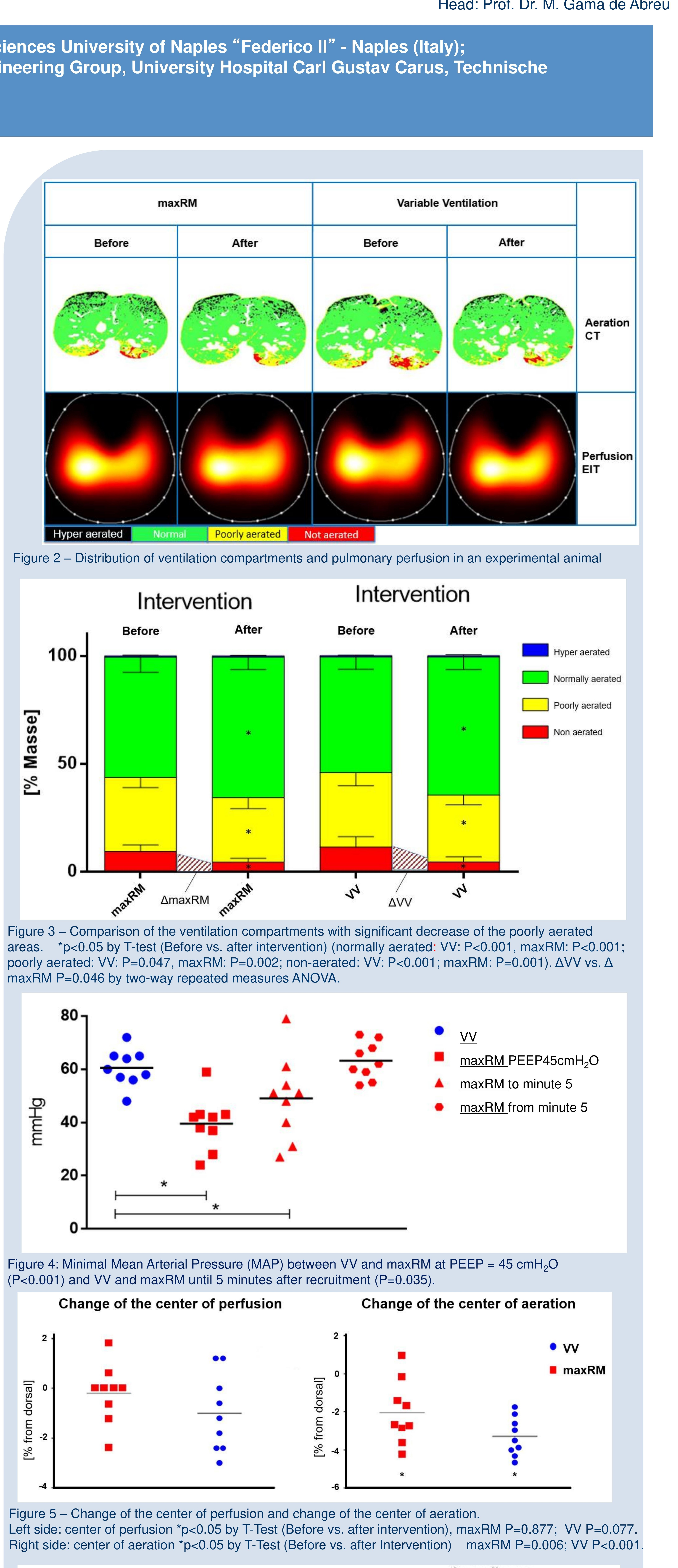

Figure 3 - Comparison of the ventilation compartments with significant decrease of the poorly aerated areas. " $p<0.05$ by T-test (Before vs. after intervention) (normally aerated: $V V: P<0.001$, maxRM: $P<0.001$ poorly aerated: $\mathrm{VV}: \mathrm{P}=0.047$, maxRM: $\mathrm{P}=0.002$; non-aerated: $\mathrm{VV}: \mathrm{P}<0.001$; maxRM: $\mathrm{P}=0.001$ ). $\Delta \mathrm{VV}$ vs. $\Delta$ $\operatorname{maxRM} P=0.046$ by two-way repeated measures ANOVA.

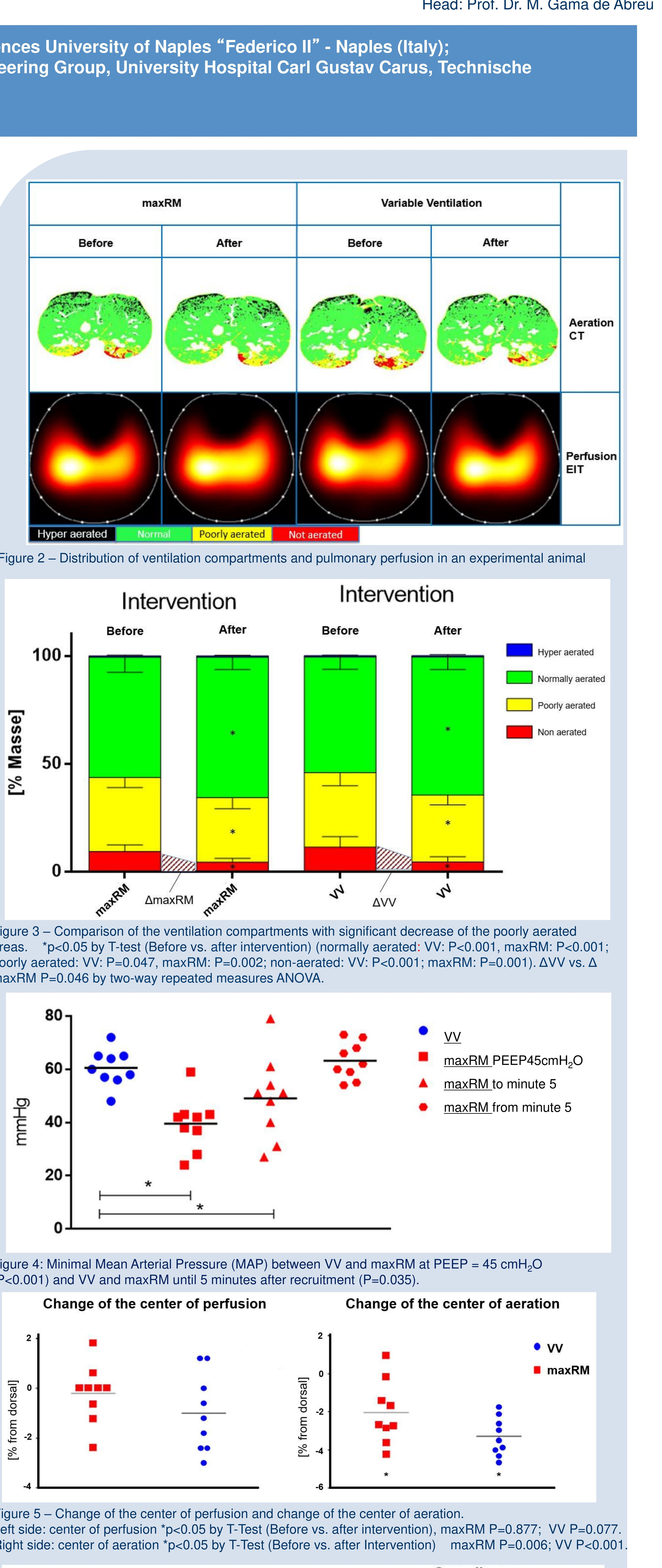

Figure 4: Minimal Mean Arterial Pressure (MAP) between VV and maxRM at PEEP $=45 \mathrm{cmH}_{2} \mathrm{O}$ $(P<0.001)$ and $V V$ and maxRM until 5 minutes after recruitment $(P=0.035)$.

Change of the center of perfusion

Change of the center of aeration

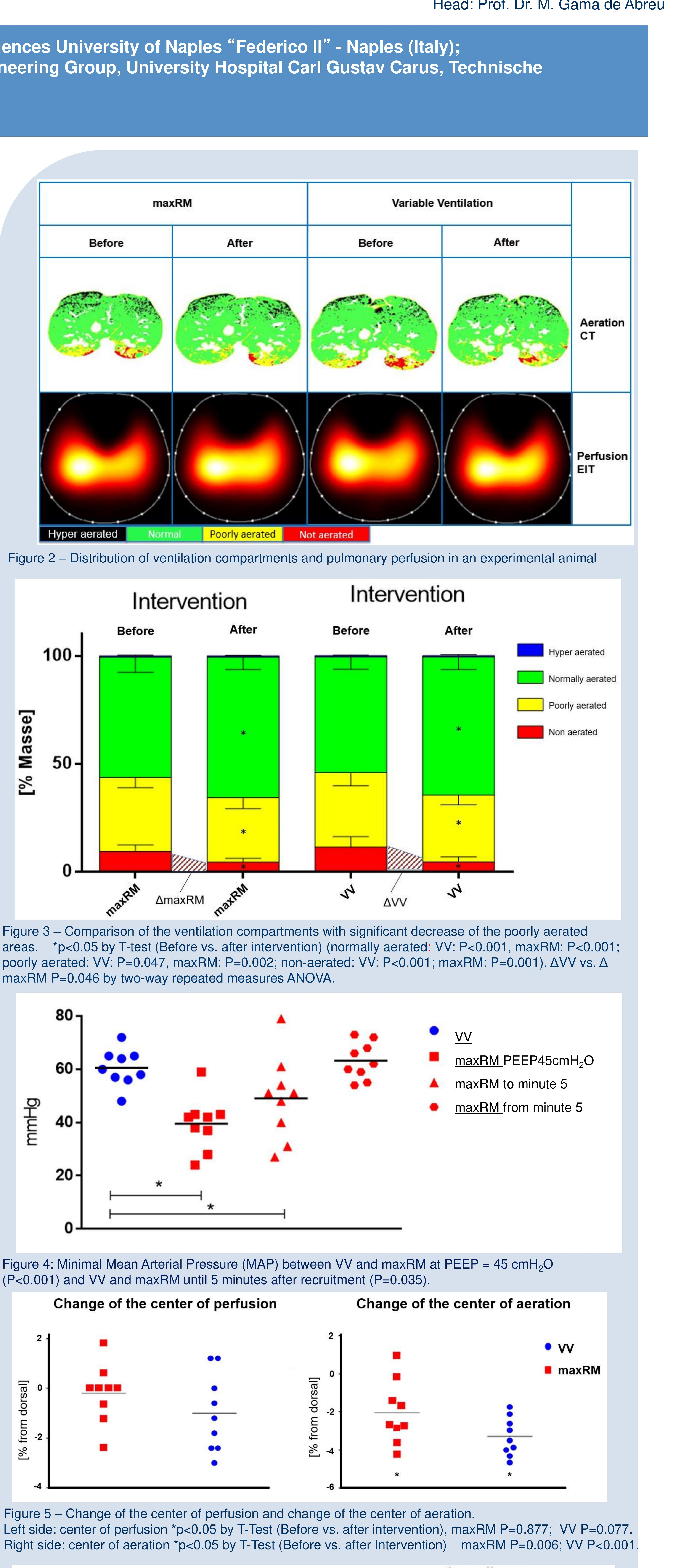

Figure 5 - Change of the center of perfusion and change of the center of aeration. . $P<0.05$ by T Test (Before 